\title{
Side Effects of CXC-Chemokine Receptor 4-Directed Endoradiotherapy with Pentixather Before Hematopoietic Stem Cell Transplantation
}

\author{
Sabine Maurer*1, Peter Herhaus*1, Romina Lippenmeyer ${ }^{2}$, Heribert Hänscheid $^{2}$, Malte Kircher ${ }^{2}$, Andreas Schirbel ${ }^{2}$, \\ H. Carlo Maurer ${ }^{3}$, Andreas K. Buck ${ }^{2}$, Hans-Jürgen Wester ${ }^{4}$, Hermann Einsele ${ }^{5}$, Götz-Ulrich Grigoleit ${ }^{\dagger 5}$, \\ Ulrich Keller ${ }^{\dagger 1,6}$, and Constantin Lapa ${ }^{\dagger 2}$ \\ ${ }^{1}$ III. Medical Department, Hematology and Medical Oncology, Technische Universität München, Munich, Germany; ${ }^{2}$ Department of \\ Nuclear Medicine, University Hospital Würzburg, Würzburg, Germany; ${ }^{3}$ II. Medical Department, Gastroenterology and Hepatology, \\ Technische Universtät München, Munich, Germany; ${ }^{4}$ Pharmaceutical Radiochemistry, Technische Universität München, Munich, \\ Germany; ${ }^{5}$ Hematology and Medical Oncology, Medical Department II, University Hospital Würzburg, Würzburg, Germany; and \\ ${ }^{6}$ Hematology, Oncology, and Tumor Immunology (Campus Benjamin Franklin), Charité-Universitätsmedizin Berlin, Berlin, Germany
}

The chemokine receptor CXC-chemokine receptor 4 (CXCR4) is a transmembrane receptor involved in survival, proliferation, and dissemination of different cancers, including hematopoietic malignancies. Relapsed or refractory hematopoietic cancers are frequently resistant to conventional therapy, and novel highly active strategies are urgently needed. CXCR4-directed endoradiotherapy constitutes a highly promising targeted therapeutic concept. Here, we investigated the adverse effects of this novel treatment approach. Methods: Twenty-two patients with heavily pretreated lymphoproliferative or myeloid malignancies were treated with ${ }^{177} \mathrm{Lu}$ - or ${ }^{90}$ Y-pentixather-a CXCR4-directed therapeutic radioligandbefore conventional conditioning therapy followed by autologous or allogeneic hematopoietic stem cell transplantation. Twenty-five CXCR4-directed endoradiotherapies were administered to those patients. Adverse events occurring between endoradiotherapy and the start of conventional conditioning therapy were retrospectively analyzed and graded for the estimation of the safety profile. Results: CXCR4-directed endoradiotherapy with pentixather showed a favorable toxicity profile. As expected, the hematopoietic system was most affected, with all subjects developing cytopenias. Except for 1 acute kidney failure, grade 3, due to tumor lysis syndrome, overall nephro- and hepatotoxicity was low. Other higher-grade adverse events were either transient and resolved or easily manageable. Conclusion: Therapy with radiolabeled pentixather appears to be well tolerated and easily applicable when preceding conventional conditioning regimens for hematopoietic stem cell transplantation.

Key Words: chemokine receptor CXCR4; endoradiotherapy; myeloma; lymphoma; leukemia

J Nucl Med 2019; 60:1399-1405

DOI: 10.2967/jnumed.118.223420

\footnotetext{
Received Nov. 14, 2018; revision accepted Feb. 20, 2019.

For correspondence or reprints contact: Ulrich Keller, Department of Hematology, Oncology, and Tumor Immunology (Campus Benjamin Franklin), Charité-Universitätsmedizin Berlin, Hindenburgdamm 30, 12203 Berlin, Germany.

E-mail: ulrich.keller@charite.de

${ }^{*}$ Contributed equally to this work.

tContributed equally to this work.

Published online Mar. 8, 2019.

COPYRIGHT (C) 2019 by the Society of Nuclear Medicine and Molecular Imaging.
}

D espite substantial advantages in the treatment of hematologic malignancies such as non-Hodgkin lymphoma, multiple myeloma, and acute myeloid leukemia, those diseases still have high relapse rates and will eventually lead to death in a large proportion of patients $(1,2)$. Novel therapeutic strategies targeting the malignant compartment but also the supporting niche are being developed with the goal of specifically reducing tumor cell survival and therapy resistance and consequently improving patient survival.

The CXC-chemokine receptor 4 (CXCR4), with its sole known ligand CXCL12 (also known as SDF-1 $\alpha$ ), is crucially involved in multiple physiologic functions such as cell growth, survival, and migration. In the hematopoietic system, CXCR4 plays a critical role in immune cell trafficking and the retention of hematopoietic stem and progenitor cells (HSPC) within their bone marrow niche. Cancer cells have been shown to hijack the physiologic functions of CXCR4, and consequently, CXCR4 overexpression has frequently been associated with a dismal prognosis (3-5). Particularly in hematologic cancers, CXCR4 is crucially involved in the crosstalk between the malignant cells and their supporting niche. Therefore, the CXCL12/CXCR4 axis is considered a highly promising target for therapy.

Because of the successful development of a radiolabeled CXCR4 ligand ( ${ }^{68} \mathrm{Ga}$-pentixafor), it is now possible to visualize CXCR4 expression in vivo by means of PET imaging $(6,7)$. Several proof-of-concept studies have shown the potential of the PET tracer ${ }^{68} \mathrm{Ga}$-pentixafor to visualize CXCR4-positive tumors, with a specific applicability for patients with hematologic diseases such as non-Hodgkin lymphoma (8), multiple myeloma $(9,10)$, and acute leukemia (11).

A theranostic approach using CXCR4-directed imaging to select patients for CXCR4-directed endoradiotherapy is enabled by the peptide pentixather. Pentixather is a modified structure of the CXCR4-directed tracer peptide pentixafor, which allows the linkage of $\beta$-emitting radionuclides $\left({ }^{177} \mathrm{Lu} ;{ }^{90} \mathrm{Y}\right)$ routinely used in clinical practice for various cancer radiotherapies (12-14). ${ }^{177} \mathrm{Lu}$-pentixather has already been proven capable of successfully eradicating CXCR4-positive tumor cells without completely and irreversibly destroying the supporting HSPC niche in a preclinical 
endoradiotherapy mouse model - a capability that is a prerequisite for the reconstitution of the hematopoietic system after therapy. Moreover, first-in-human experiences of CXCR4-directed endoradiotherapy with pentixather in patients with advanced-stage multiple myeloma, acute myeloid leukemia, and non-Hodgkin lymphoma have shown the feasibility of this therapy in combination with high-dose chemotherapy and autologous or allogeneic hematopoietic stem cell transplantation (HSCT) $(10,15-17)$. However, because of the high expression levels of CXCR4 on HSPCs and its crucial function in their regulation, the safety of CXCR4-directed endoradiotherapy has to be further studied.

Here, we report on the toxicity profile of CXCR4-directed endoradiotherapy with ${ }^{177} \mathrm{Lu}$ - and ${ }^{90}$ Y-pentixather. For this retrospective analysis, we analyzed data from 25 CXCR4-directed therapies with pentixather that were administered within individual therapy approaches on a compassionate-use basis in 22 patients. By reporting the side effects of this therapy in a substantial patient group, this investigation provides crucial information for the design and implementation of future prospective clinical studies.

\section{MATERIALS AND METHODS}

\section{Patients}

Twenty-two patients with advanced lymphoproliferative and myeloid cancers were treated and studied from 2014 to 2016 at the University Hospital Würzburg. All patients were heavily pretreated and had no option for further standard therapies.

Given the lack of alternative treatment options, and in view of the documented CXCR4 expression (confirmed in vivo by ${ }^{68} \mathrm{Ga}$-pentixafor PET imaging) and the availability of an HSCT donor or an autologous graft, an interdisciplinary board of specialists opted for CXCR4targeted endoradiotherapy combined with high-dose conditioning chemotherapy and autologous or allogeneic HSCT. The decision on combination of pentixather with a second radiopharmaceutical was based on patient and disease characteristics and the type of transplantation and subsequently performed as suggested within the interdisciplinary board. The clinical ethics committee of the University Hospital Würzburg (responsible internal review board) approved individual treatments on a compassionate-use basis (German Drug Act, $\S 13,2 b)$. All subjects gave written informed consent before receiving the therapy.

The treatment-related data of some patients have been previously described in feasibility studies of CXCR4-directed endoradiotherapy with pentixather (15-17). Those data have thus not been analyzed in a structured retrospective manner before now.

Rationale for Repeated Pentixather Treatment in 2 Patients. One patient with very advanced multiple myeloma received 3 cycles of CXCR-directed endoradiotherapy, of which 2 were combined with autologous HSCT and 1 with allogeneic HSCT. The repeated treatment in this patient was administered because of an initial response to CXCR4-directed endoradiotherapy and the lack of further standard treatment options. In another patient with multiple myeloma, 2 cycles of CXCR4-directed endoradiotherapy were performed: 1 cycle before autologous and 1 cycle before allogeneic HSCT. Treatment response to conditioning therapy combined with pentixather and autologous HSCT was obtained, and CXCR4-directed endoradiotherapy was therefore later incorporated into the conditioning regimen before allogeneic HSCT.

Pretherapeutic Dosimetry. One or $2 \mathrm{~d}$ after the administration of the tracer activity, which was infused without any nephroprotective medication, activity concentrations were measured by quantitative SPECT/CT in kidneys, liver, spleen, bone marrow, and, if possible, malignant tissues. Serial planar whole-body images were acquired over
3-5 d to deduce the activity kinetics approximated by biexponential decay functions in the listed tissues. The decay functions were normalized to the activity concentrations measured by SPECT/CT and integrated to determine the absorbed doses per activity administered. The maximum absorbed doses were deduced for kidneys, bone marrow, and malignant tissues from the highest activity concentrations measured in contiguous volumes of $1 \mathrm{~cm}^{3}$. For liver and spleen, mean absorbed doses were measured in large representative volumes within the organs.

All scintigraphic images were acquired with a $20 \%$ energy window at $208 \mathrm{keV}$ using dual-head $\gamma$-cameras (Siemens Symbia E or Symbia T2) equipped with medium-energy collimators. Whole-body images were acquired at $10 \mathrm{~cm} / \mathrm{min}$ and a matrix of $256 \times 1,024$. SPECT was measured with a matrix of $128 \times 128$, a voxel size of $4.8 \mathrm{~mm}(0.11$ $\mathrm{cm}^{3}$ ), and $2 \times 60$ frames at $30 \mathrm{~s}$ per projection with a $3^{\circ}$ angular step. SPECT data were reconstructed iteratively by 3 -dimensional orderedsubsets expectation maximization (6 subsets, 6 iterations) with corrections for scatter and attenuation after a low-dose CT transmission scan.

\section{Dosimetry}

The dosage of activity for endoradiotherapy was based on the result of a pretherapeutic dosimetry study with a tracer activity of ${ }^{177} \mathrm{Lu}-$ pentixather in each patient.

In patients treated with ${ }^{177} \mathrm{Lu}$-pentixather, the measurements were repeated with reduced acquisition durations to assess the actually absorbed doses. Because of the inherent problems and uncertainties of dose determination with pure $\beta$-emitters, no dosimetry was performed after administration of ${ }^{90} \mathrm{Y}$. Therapeutic absorbed doses per unit activity of ${ }^{90}$ Y-pentixather were estimated by recalculating the biexponential decay functions measured with ${ }^{177} \mathrm{Lu}$ in the tracer study to the shorter half-life of the ${ }^{90} \mathrm{Y}$.

\section{Therapy}

The administered endoradiotherapy activities for myeloablation were chosen to target at a 23-Gy maximum absorbed dose to the kidneys. The specific absorbed dose determined from the tracer study for the $1-\mathrm{cm}^{3}$ volume with the highest activity concentration within the kidneys was used to calculate the required activity. To reduce trapping of peptides in renal tubules and thus toxicity, amino acid solution $(25 \mathrm{~g}$ of L-arginine and $25 \mathrm{~g}$ of L-lysine diluted in $2 \mathrm{~L}$ of normal saline) was coinfused for myeloablative therapy as recommended for peptide receptor radionuclide therapy (18).

\section{Assessment of Adverse Events (AEs)}

Vital signs, complete blood count, and serum chemistry, including values corresponding to the kidney and liver function as well as electrolytes, were documented daily starting the day of the infusion of the radionuclide. In addition to the lab work analysis, a clinical assessment (gastrointestinal, cardiac, renal, pulmonary, nervous system, skin, and for possible signs of infection) was performed daily according to the standards of the transplant unit. AEs that occurred from the day of the radionuclide infusion to the day of the start of the conventional conditioning regimen were included into the retrospective analysis. AEs occurring after the beginning of the conditioning regimens were considered to be due to chemotherapy and the transplantation itself and were not included in this analysis.

All AEs were graded according to the National Cancer Institute Common Terminology Criteria for Adverse Events, version 4.03.

\section{Statistical Analysis}

All statistical tests were performed using GraphPad Prism (GraphPad Software). $P$ values of less than 0.05 were considered statistically significant. Quantitative values were expressed as mean $\pm \mathrm{SD}$. The Student $t$ test was used to compare quantitative data between cohorts. 
Because of the retrospective nature of the analysis, the sample size was based on the number of consecutive patients treated with the respective endoradiotherapy in the specified time frame.

\section{RESULTS}

\section{Patient Characteristics}

In total, 25 CXCR4-directed endoradiotherapies with pentixather were administered to 22 patients with advanced blood cancers before conventional conditioning therapy followed by autologous or allogeneic HSCT. The median age was $54 \mathrm{y}$, with a range from 31 to $68 \mathrm{y}$. Ten patients presented with multiple myeloma, 6 with diffuse large B-cell lymphoma, and 4 with acute myeloid leukemia. One patient each had a diagnosis of mantle cell lymphoma and T-cell prolymphocytic leukemia. All patients were heavily pretreated, having received at least 2 standard therapy regimens and a median of 4 previous therapy lines (standard or experimental). Over $80 \%$ of the patients had a previous autologous or allogeneic HSCT. Detailed patient characteristics are displayed in Table 1.

\section{Dosimetry and Treatment Characteristics}

Activities of $206 \pm 10 \mathrm{MBq}$ of ${ }^{177} \mathrm{Lu}$-pentixather were administered for pretherapeutic dosimetry. The kidneys were the doselimiting organ for all patients receiving treatment for myeloablative therapy. Activity kinetics in the kidneys was generally biexponential, with a negative short-lived component indicating further accumulation early after the activity administration, and a dominant long-lived component. The effective half-life of the dominant component was $37 \pm 12 \mathrm{~h}$ with 1 outlier at $97 \mathrm{~h}$, which is

TABLE 1

Patient Characteristics

\begin{tabular}{lc}
\hline \multicolumn{1}{c}{ Characteristic } & Data \\
\hline Sex $(n)$ & $12(55 \%)$ \\
\hline Male & $10(45 \%)$ \\
\hline Female & \\
Age $(y)$ & 54 \\
\hline Median & $31-68$ \\
\hline Range & \\
Diagnosis $(n)$ & $4(18 \%)$ \\
\hline Acute myeloid leukemia & $10(45 \%)$ \\
\hline Multiple myeloma & $6(27 \%)$ \\
\hline DLBCL & $1(5 \%)$ \\
\hline MCL & $1(5 \%)$ \\
\hline T-PLL & \\
\hline Previous treatment lines $(n)$ & 4 \\
\hline Median & $2-9$ \\
\hline Range & $18(82 \%)$ \\
\hline Patients with previous transplantation $(n)$ & $12(55 \%)$ \\
\hline Autologous & $6(27 \%)$ \\
\hline Allogenic &
\end{tabular}

$\mathrm{DLBCL}=$ diffuse large B-cell lymphoma; $\mathrm{MCL}=$ mantle cell lymphoma; T-PLL = T-cell prolymphocytic leukemia. slightly shorter than typically observed in octreotide endoradiotherapy (19-21). The renal absorbed dose per unit administered activity was $1.6 \pm 0.7 \mathrm{~Gy} / \mathrm{GBq}{ }^{177} \mathrm{Lu}$-pentixather without nephroprotection, which is slightly higher than in octreotide endoradiotherapy (22). The nephroprotective medication reduced the therapeutic maximum kidney dose per administered activity in the 6 patients treated with ${ }^{177} \mathrm{Lu}$-pentixather to $64 \% \pm 13 \%$ of the values measured in pretherapeutic dosimetry. Recalculation of the kidney doses for the therapy with ${ }^{90}$ Y-pentixather with protective medication resulted in $4.0 \pm 1.7 \mathrm{~Gy} / \mathrm{GBq}$. Slight mean decreases in specific absorbed doses during therapy were also observed for the liver $(86 \% \pm 15 \%)$, the spleen $(88 \% \pm 8 \%)$, and extramedullary lesions $(82 \% \pm 30 \%)$ in the patients treated with ${ }^{177} \mathrm{Lu}$-pentixather (Supplemental Table 1; supplemental materials are available at http://jnm.snmjournals.org). In these patients, the mean effective half-life of the therapeutic activity in the bone marrow was $115 \pm 14 \mathrm{~h}$; the expected effective halflife for ${ }^{90} \mathrm{Y}$ in the marrow would have been $55 \pm 3 \mathrm{~h}$. A representative pretherapeutic PET/CT scan with ${ }^{68} \mathrm{Ga}$-pentixafor, as well as the dosimetry data from 1 patient with an extramedullary acute myeloid leukemia, is shown in Figure 1.

According to the pretherapeutic dosimetry and with a maximum tolerated kidney dose of $23 \mathrm{~Gy}$, patients were treated for myeloablation by intravenous infusion of 7.6-23.5 GBq of ${ }^{177} \mathrm{Lu}$-pentixather (6 therapies) or $2.4-8.4 \mathrm{GBq}$ of ${ }^{90}$ Y-pentixather (19 therapies). CXCR4-directed endoradiotherapy with ${ }^{90}$ Y-pentixather was further combined with ${ }^{188} \mathrm{Re}-\mathrm{CD} 66$ in 6 patients and ${ }^{90}$ Y-ibritumomab tiuxetan (Zevalin; Spectrum Pharmaceuticals) and ${ }^{153}$ Sa-ethylenediamine tetra(methylene phosphonic acid) (EDTMP) in 1 patient each (Table 2; Supplemental Table 2). The expected kidney doses from the additional therapies were considered in the dosage of pentixather. The actually administered doses from therapy with pentixather in the cubic centimeter with the highest activity concentration in the kidneys were measured to be $13.0 \pm 1.9 \mathrm{~Gy}$ (range, 10.5-16.0 Gy) after ${ }^{177} \mathrm{Lu}$ and, taking the protective medication during therapy into account, estimated to be $16.4 \pm 3.6 \mathrm{~Gy}$ (range, 11.2-22.2 Gy) after ${ }^{90} \mathrm{Y}$.

\section{AEs}

In total, 340 AEs were detected after the 25 pentixather treatment courses. Among those, 86 events $(25.3 \%)$ showed a severity of grade 3 or higher. One patient developed acute tumor lysis syndrome with grade 3 acute kidney failure that led to discontinuation of the further treatment plan (Table 3). The therapy regimen (endoradiotherapy, conditioning chemotherapy, HSCT) could be finished as intended in all 24 of the other CXCR4-directed treatment courses.

The most common AEs were expectedly seen in the hematopoietic system. During all treatment courses, patients developed anemia, after 17 of 25 courses bearing grade 3 or grade 4 toxicity. All patients developed neutropenia and thrombocytopenia, which were of grade 3 or 4 in at least 20 of 25 treatment courses, irrespective of the administered radionuclide $\left({ }^{177} \mathrm{Lu}\right.$ or ${ }^{90} \mathrm{Y}$ ) (Table 4; Supplemental Table 3; Fig. 2).

Sites of elimination and excretion of the radiopharmaceutical include kidney and liver. Thus, the impact on renal and hepatic function was of special interest. Notably, only during 2 of 25 treatment courses did patients present with acute kidney failure. During 1 treatment course, a patient developed grade 3 kidney failure in the context of tumor lysis syndrome mentioned above. 


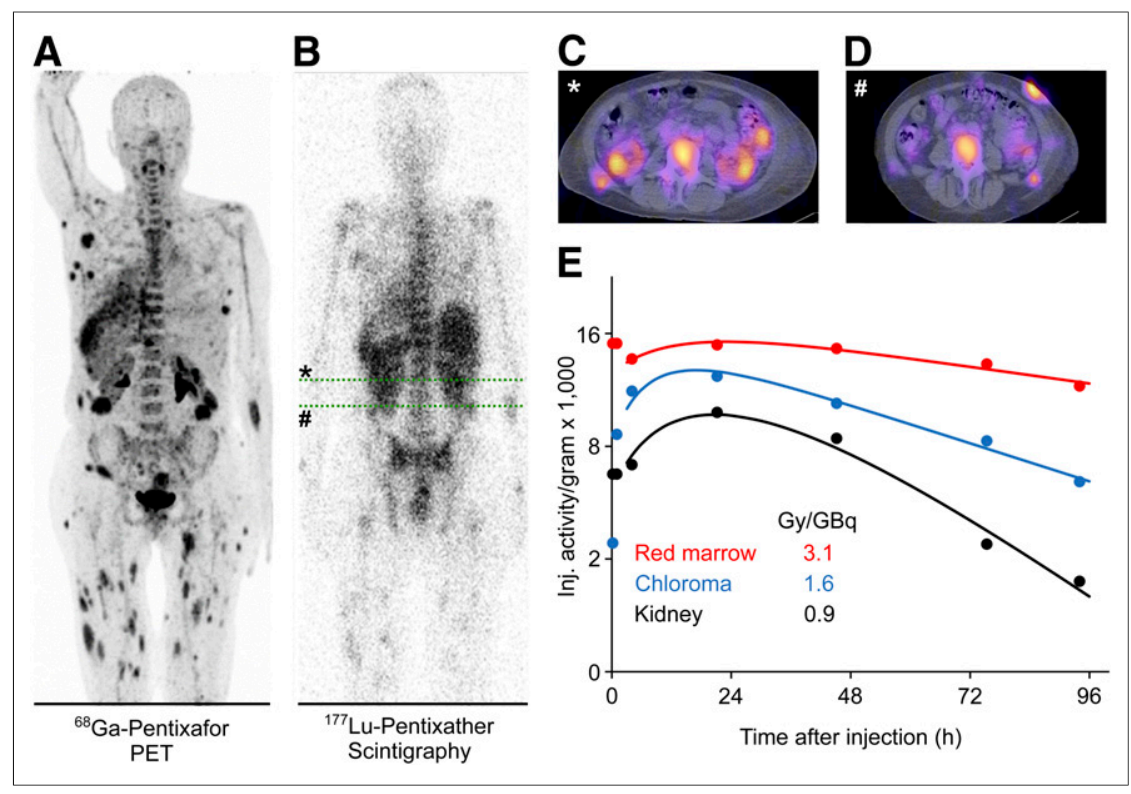

FIGURE 1. Pretherapeutic ${ }^{68} \mathrm{Ga}$-pentixafor PET and dosimetry with ${ }^{177} \mathrm{Lu}$-pentixather in representative patient with extramedullary acute myeloid leukemia. (A) Maximum-intensity projection of ${ }^{68} \mathrm{Ga}$-pentixafor PET (89 MBq of ${ }^{68} \mathrm{Ga}$-pentixafor, acquisition $1 \mathrm{~h}$ after injection). (B) Planar whole-body scintigraphy (204 MBq of ${ }^{177}$ Lu-pentixather, acquisition $2 \mathrm{~d}$ after injection). Concordant tracer accumulation is seen in BM, as well as multiple extramedullary acute myeloid leukemia lesions. (C and D) Sufficient CXCR4 expression can also be determined in transaxial SPECT/CT (representative images of abdomen [green lines in B)]. (E) Time-activity curves (up to $96 \mathrm{~h}$ after injection) demonstrating highest achievable dose per injected activity for red marrow.

In $58 \%$ of the treatment courses, we observed an elevation of transaminases, and in $23 \%$, bilirubin levels increased. Importantly, those changes were not severe (grade 1-2), and there was no higher-grade toxicity or limitation of the hepatic synthesis activity (e.g., disruption of plasmatic coagulation) (Supplemental Table 4; Table 4).

Fitting the high percentage of neutropenia after application of the radiopeptide, and as expected in such a heavily pretreated patient cohort with hematopoietic cancers, a substantial rate of infections, including grade 3 or grade 4 infections (sepsis), occurred after 9 of the 25 treatment courses (36\%). Notably, all these patients recovered after preemptive or targeted antibiotic treatment and could proceed to the conditioning chemotherapy and HSCT (Supplemental Table 5).

Other AEs, such as electrolyte disorders (Supplemental Table 6), gastrointestinal toxicities (Supplemental Table 7), cardiovascular side effects (Supplemental Table 8), toxicities of the nervous

TABLE 2

Overview of Radionuclides, Peptides, and Antibodies

\begin{tabular}{lc}
\hline \multicolumn{1}{c}{ Radionuclide } & $n$ \\
\hline${ }^{177}$ Lu-CPCR4 & $6(24 \%)$ \\
${ }^{90}$ Y-CPCR4 & $11(44 \%)$ \\
${ }^{90}$-CPCR4 $+{ }^{188}$ Re-CD66 & $6(24 \%)$ \\
${ }^{90}$ Y-CPCR4 $+{ }^{90}$ Y-ibritumomab tiuxetan & $1(4 \%)$ \\
${ }^{90}$ Y-CPCR4 $+{ }^{153}$ Sm-EDTMP & $1(4 \%)$ \\
\hline
\end{tabular}

system (Supplemental Table 9), or general disorders (Supplemental Table 10), were in general self-limiting or manageable and did not delay further therapy.

\section{Characteristics of Further Therapy}

After treatment with pentixather, all but 1 patient underwent subsequent conditioning/high-dose chemotherapy with either autologous or allogeneic HSCT (Table 5). As stated above, treatment with ${ }^{177} \mathrm{Lu}$ pentixather, as well as ${ }^{90}$ Y-pentixather, led to grade 3 or grade 4 cytopenias and thereby to the assumptive reduction of tumor cell burden in the hematopoietic niche (Table 4; Supplemental Table 2; Fig. 2).

Conventional chemotherapy and subsequent HSCT were first started when radiation was decayed, as the transplanted HSPCs should not be exposed to radiation-associated toxicity. It was therefore not unexpectedbecause of the shorter half-life of ${ }^{90} \mathrm{Y}$ (2.7 d, compared with $6.7 \mathrm{~d}$ for ${ }^{177} \mathrm{Lu}$ ) that the median time from CXCR4-directed endoradiotherapy to the beginning of conventional conditioning therapy was significantly shorter in patients who received ${ }^{90}$ Y-pentixather than in those who received ${ }^{177} \mathrm{Lu}$-pentixather (Fig. 3).

Engraftment of HSPC, defined by leukocyte and platelet engraftment, was not impaired by endoradiotherapy with pentixather. Leukocyte engraftment occurred on day 11.4 (mean; range, days 9-14) after autologous and on day 12.2 (mean; range, days 10-20) after allogeneic HSCT. On day 14 (mean; range, days 9-18) after autologous and on day 13.25 (mean; range, days 10-16) after allogeneic HSCT, platelet engraftment was reached (Table 5). Because of the heavy pretreatment of the patient collective, often with active disease and poor general condition, 10 patients died from neutropenic sepsis or tumor progression before complete engraftment.

\section{DISCUSSION}

The toxicity profile obtained from this retrospective analysis clearly demonstrates the feasibility of CXCR4-directed endoradiotherapy with pentixather, when combined with conventional conditioning regimens. Because of the high rates of hematologic toxicities, we currently assume that this therapy can successfully be applied only embedded in an HSCT concept.

Because of the relatively high CXCR4 expression within the HSPC compartment, the most severe adverse effects could be

TABLE 3

Overview of AEs

\begin{tabular}{lc}
\hline \multicolumn{1}{c}{ Event } & $n$ \\
\hline Any AE & 340 \\
Any AE of grade $\geq 3$ & 86 \\
AE resulting in treatment discontinuation & $1(4 \%)$ \\
\hline
\end{tabular}


TABLE 4

Hematologic Toxicities

\begin{tabular}{cc}
\hline AE & $n$ \\
\hline Anemia & \\
\hline Any grade & $25(100 \%)$ \\
Grade $\geq 3$ & $17(68 \%)$ \\
Thrombopenia & \\
Any grade & $25(100 \%)$ \\
Grade $\geq 3$ & $21(84 \%)$ \\
Neutropenia & \\
Any grade & $24(96 \%)$ \\
Grade $\geq 3$ & $20(80 \%)$ \\
Coagulopathy & \\
Any grade & $7(28 \%)$ \\
Grade $\geq 3$ & $0(0 \%)$ \\
\hline
\end{tabular}

attributed to the hematopoietic system, with approximately $70 \%$ of higher-grade AEs involving cytopenias. Apart from 1 patient who developed tumor lysis syndrome that subsequently led to discontinuation of therapy, no further deaths could directly be attributed to endoradiotherapy. It is important to consider that hematotoxicity could reflect a sufficient eradication of the HSPC compartment, which should be studied in subsequent trials by bone marrow biopsies after endoradiotherapy and before further conditioning.

Higher doses of total-body irradiation before HSCT go along with superior remission rates. However, because of the severe nonhematologic side effects of unselective irradiation, which often lead to an unacceptable rate of treatment-related mortality, those conditioning regimens are restricted to young and healthy patients (23). CXCR4-directed endoradiotherapy might have the potential to circumvent the nonhematologic side effects of total-body irradiation and therefore translate the high remission rate attributed to radiation into an increased rate of overall survival. Furthermore, such endoradiotherapy provides the possibility to be used after previous conditioning with totalbody irradiation.

Although the eradication of the tumor-supporting niche, caused by crossfire effects when using $\beta$-emitters, is presumed a major point of action in CXCR4-directed endoradiotherapy, destruction of the stem cell niche could bear an incalculable potential risk. An impaired support of the transplanted HSPCs may lead to engraftment failure and long-lasting cytopenias, which have been associated with high mortality rates (24). Recent work in a preclinical mouse model, however, showed that mesenchymal stem cells, or stromal cells, an important component of the bone marrow stem cell niche, preserve their ability to support HSPCs after CXCR4directed endoradiotherapy with ${ }^{177} \mathrm{Lu}$-pentixather (16). Those observations are mirrored by the unimpaired leukocyte and platelet engraftment after therapy with both ${ }^{90} \mathrm{Y}$ - and ${ }^{177} \mathrm{Lu}$-labeled pentixather and subsequent HSCT in the herein studied patient population, which compared well with mean leukocyte engraftment of $16.4 \mathrm{~d}$ and mean platelet engraftment of $13 \mathrm{~d}$ after allogeneic HSCT (25).

Because of tracer elimination through the liver and, most importantly, through renal excretion, toxicity to these organs limits the maximal administered activity of radionuclide-conjugated pentixather. The activity dosage in our patients was conservative insofar as no kidney protection medication was administered in the tracer study and the limit of 23 Gy was not applied to the mean kidney dose but to the $1-\mathrm{cm}^{3}$ partial volume with the maximum activity concentration. Therefore, it is of great significance that this retrospective analysis revealed no grade 3 or higher acute liver toxicity in the 25 endoradiotherapy courses. The 1 case of acute kidney failure occurred in a patient with tumor lysis syndrome. The specific absorbed dose in the $1-\mathrm{cm}^{3}$ volume with the highest activity concentration in the kidneys was determined from the tracer study to be $7.0 \mathrm{~Gy} / \mathrm{GBq}{ }^{90}$ Y-pentixather; the patient received $2.62 \mathrm{GBq}$, corresponding to an estimated dose of 18.3 Gy. It is speculative that the observed endoradiotherapy-mediated tumor lysis in combination with acute radiation-associated toxicity contributed to the further clinical course.

Apart from liver and renal toxicity, most other AEs were transient and self-limiting without further intervention, except for the high rates of infections observed after endoradiotherapy. These are most likely attributable to the high rates of leuko- and neutropenia and were usually manageable with antibiotic treatment. Importantly, these infectious complications underscore the importance of aiming for a short period of neutropenia and a short interval to conventional conditioning and HSCT, as septic events during this time are frequent causes of treatment-related mortality in the setting of HSCT (26). Very importantly, this analysis disclosed a significantly and clinically relevant shorter interval between endoradiotherapy and the beginning of the conventional conditioning therapy for ${ }^{90}$ Y-pentixather than for ${ }^{177} \mathrm{Lu}$-pentixather.

Within this analysis, we purposely chose to limit the report of side effects to the interval between endoradiotherapy and the start of conventional therapy to get an initial impression of acute side effects of CXCR4-directed endoradiotherapy. Therefore, no definitive conclusion can be drawn on long-term toxicities of CXCR4-directed endoradiotherapy with pentixather or on additive or synergistic effects in the combination with conventional therapies. Studies on other radiolabeled peptides, such as ${ }^{177}$ Lu-DOTATATE, have shown a slightly
FIGURE 2. Cytopenias relative to baseline after CXCR4-directed endoradiotherapy. Depicted is reduction of hemoglobin, leukocyte, granulocyte, and platelet values in percentage from baseline after respective pentixather therapy $\left({ }^{177} \mathrm{Lu}\right.$ or $\left.{ }^{90} \mathrm{Y}\right)$ before start of conventional conditioning therapy. Each dot represents value of 1 therapy. $P$ values were calculated using $t$ test. 
TABLE 5

Transplant Characteristics

\begin{tabular}{lc}
\hline \multicolumn{1}{c}{ Characteristic } & Data \\
\hline Transplantation type $(n)$ & $10(40 \%)$ \\
\hline Autologous & $14(56 \%)$ \\
\hline Allogenic & \\
\hline $\begin{array}{l}\text { Mean time to engraftment, } \\
\text { leukocytes (d) }\end{array}$ & 11.4 (range, 9-14) \\
$\quad$ Autologous & 12.2 (range, 10-20) \\
\hline Allogenic & \\
\hline $\begin{array}{l}\text { Mean time to engraftment, } \\
\text { platelets (d) }\end{array}$ & 14 (range, 9-18) \\
\hline Autologous & 13.25 (range, 10-16) \\
\hline Allogenic &
\end{tabular}

increased risk of peptide receptor radionuclide therapy for the development of myelodysplastic syndromes or acute myeloid leukemia $(13,27)$. These long-term toxicities concerning the hematopoietic system are most probably caused by radiation-associated toxicity on the HSPCs. Such toxicities might, however, be bypassed by the integration of CXCR4-directed endoradiotherapy in an HSCT concept.

Although this retrospective analysis in a substantial number of patients provides a fairly detailed toxicity profile for CXCR4directed endoradiotherapy and demonstrates its feasibility and points to putative effectiveness of this therapy, there are evident limitations. First, because of the retrospective analysis of the data and the lack of prospective, coordinated data collection as obtained within a controlled study, some toxicity data of clinical relevance could have been missed. Second, the patient collective described is very heterogeneous, and previous therapies and conditioning regimens have not been consistent. Moreover, some of the observed toxicities might have been caused by the

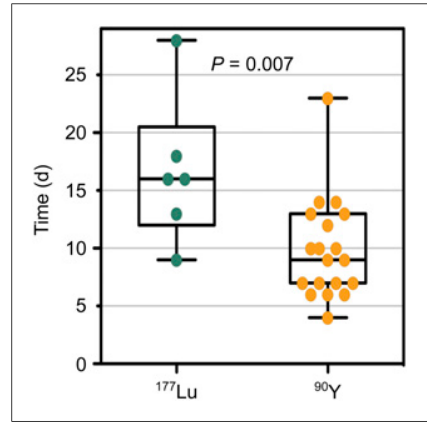

FIGURE 3. Interval between CXCR4-directed endoradiotherapy and start of conventional conditioning therapy. Depicted are days between application of either ${ }^{177} \mathrm{Lu}-$ pentixather or ${ }^{90} \mathrm{Y}$-pentixather (half-life of ${ }^{177}$ Lu vs. ${ }^{90} \mathrm{Y}, 6.7$ vs. $2.7 \mathrm{~d}$ ) until start of conventional conditioning chemotherapy. Each dot represents value of 1 therapy. $P$ values were calculated using $t$ test. administration of other radionuclide-conjugated vec-

with HSCT and to target the stem cell niche. With a side effect profile pointing toward grade 3-4 AEs within the hematopoietic compartment, where tumor burden was high and where this effect is anticipated and desirable, the use of radiolabeled pentixather represents a promising treatment option in heavily pretreated patients with advanced lymphoproliferative and myeloid CXCR4-positive cancers. Further investigations regarding safety and efficacy should be performed in the context of prospective phase I/II studies.

\section{DISCLOSURE}

Ulrich Keller received support from Deutsche Forschungsgemeinschaft (SFB 824 project C3, SFB 1335 project P3) and by Deutsche Krebshilfe (project 111944) and is further funded by Stiftung Charité. No other potential conflict of interest relevant to this article was reported.

\section{ACKNOWLEDGMENTS}

We thank all members of the Hematology/Oncology and the Nuclear Medicine clinical teams for excellent patient care and for support in documentation and obtaining data. We in particular thank Daina Meys for her help with data documentation.

\section{KEY POINTS}

QUESTION: CXCR4-directed endoradiotherapy in patients with advanced hematopoietic malignancies: What are the specifications and dimensions of the acute side effects of this novel treatment approach?

PERTINENT FINDINGS: This retrospective analysis of 22 patients receiving CXCR4-directed endoradiotherapy with radiolabeled pentixather within hematopoietic stem cell transplantation protocols identified a manageable toxicity profile expected for CXCR4 targeting.

IMPLICATIONS FOR PATIENT CARE: Pentixather endoradiotherapy seems well tolerated and was easily applicable. This treatment warrants further prospective clinical evaluation for heavily pretreated patients with CXCR4-positive disease.

\section{REFERENCES}

${ }^{90}$ Y-ibritumomab tiuxetan, and ${ }^{153}$ Sa-EDTMP. A prospective clinical phase I/II trial is therefore urgently needed to clearly determine the toxicity and effectiveness of CXCR4-directed endoradiotherapy with pentixather and will be conducted in the COLPRIT trial (EudraCT 2015-001817-28).

\section{CONCLUSION}

CXCR4-directed endoradiotherapy with pentixather could be a safe and easily applicable way to further enhance the antitumor effect of high-dose chemotherapy and conditioning regimens in combination
1. Lenz G, Staudt LM. Aggressive lymphomas. N Engl J Med. 2010;362:14171429 .

2. Hiddemann W, Cheson BD. How we manage follicular lymphoma. Leukemia. 2014;28:1388-1395.

3. Peled A, Tavor S. Role of CXCR4 in the pathogenesis of acute myeloid leukemia. Theranostics. 2013;3:34-39.

4. Ratajczak MZ, Serwin K, Schneider G. Innate immunity derived factors as external modulators of the CXCL12-CXCR4 axis and their role in stem cell homing and mobilization. Theranostics. 2013;3:3-10.

5. Domanska UM, Kruizinga RC, Nagengast WB, et al. A review on CXCR4/ CXCL12 axis in oncology: no place to hide. Eur J Cancer. 2013;49:219-230.

6. Demmer O, Gourni E, Schumacher U, Kessler H, Wester HJ. PET imaging of CXCR4 receptors in cancer by a new optimized ligand. ChemMedChem. 2011; 6:1789-1791

7. Gourni E, Demmer O, Schottelius M, et al. PET of CXCR4 expression by a ${ }^{68} \mathrm{Ga}$-labeled highly specific targeted contrast agent. J Nucl Med. 2011;52: 1803-1810.

8. Wester HJ, Keller U, Schottelius M, et al. Disclosing the CXCR4 expression in lymphoproliferative diseases by targeted molecular imaging. Theranostics.

9. Philipp-Abbrederis K, Herrmann K, Knop S, et al. In vivo molecular imaging of chemokine receptor CXCR4 expression in patients with advanced multiple myeloma. EMBO Mol Med. 2015;7:477-487. 2015;5:618-630. 
10. Lapa C, Schreder M, Schirbel A, et al. $\left[{ }^{68} \mathrm{Ga}\right]$ pentixafor-PET/CT for imaging of chemokine receptor CXCR4 expression in multiple myeloma: comparison to $\left[{ }^{18}\right.$ F]FDG and laboratory values. Theranostics. 2017;7:205-212.

11. Herhaus P, Habringer S, Philipp-Abbrederis K, et al. Targeted positron emission tomography imaging of CXCR4 expression in patients with acute myeloid leukemia. Haematologica. 2016;101:932-940.

12. Schottelius M, Osl T, Poschenrieder A, et al. $\left[{ }^{177} \mathrm{Lu}\right]$ pentixather: comprehensive preclinical characterization of a first CXCR4-directed endoradiotherapeutic agent. Theranostics. 2017;7:2350-2362.

13. Strosberg J, El-Haddad G, Wolin E, et al. Phase 3 trial of ${ }^{177} \mathrm{Lu}$-dotatate for midgut neuroendocrine tumors. N Engl J Med. 2017;376:125-135.

14. Imhof A, Brunner P, Marincek N, et al. Response, survival, and long-term toxicity after therapy with the radiolabeled somatostatin analogue $\left[{ }^{90} \mathrm{Y}\right.$ DOTA]-TOC in metastasized neuroendocrine cancers. J Clin Oncol. 2011;29: 2416-2423.

15. Herrmann K, Schottelius M, Lapa C, et al. First-in-human experience of CXCR4-directed endoradiotherapy with ${ }^{177} \mathrm{Lu}$ - and ${ }^{90}$ Y-labeled pentixather in advanced-stage multiple myeloma with extensive intra- and extramedullary disease. J Nucl Med. 2016;57:248-251.

16. Habringer S, Lapa C, Herhaus P, et al. Dual targeting of acute leukemia and supporting niche by CXCR4-directed theranostics. Theranostics. 2018; 8:369-383.

17. Lapa C, Hanscheid H, Kircher M, et al. Feasibility of CXCR4-directed radioligand therapy in advanced diffuse large B cell lymphoma. J Nucl Med. 2019; 60:60-64.

18. Bodei L, Mueller-Brand J, Baum RP, et al. The joint IAEA, EANM, and SNMMI practical guidance on peptide receptor radionuclide therapy (PRRNT) in neuroendocrine tumours. Eur J Nucl Med Mol Imaging. 2013;40:800-816.
19. Heikkonen J, Maenpaa H, Hippelainen E, Reijonen V, Tenhunen M. Effect of calculation method on kidney dosimetry in ${ }^{177} \mathrm{Lu}$-octreotate treatment. Acta Oncol. 2016;55:1069-1076.

20. Garske U, Sandstrom M, Johansson S, et al. Minor changes in effective half-life during fractionated ${ }^{177} \mathrm{Lu}$-octreotate therapy. Acta Oncol. 2012;51:86-96.

21. Hänscheid H, Lapa C, Buck AK, Lassmann M, Werner RA. Dose mapping after endoradiotherapy with ${ }^{177} \mathrm{Lu}$-DOTATATE/DOTATOC by a single measurement after 4 days. J Nucl Med. 2018;59:75-81.

22. Chalkia MT, Stefanoyiannis AP, Chatziioannou SN, Round WH, Efstathopoulos EP, Nikiforidis GC. Patient-specific dosimetry in peptide receptor radionuclide therapy: a clinical review. Australas Phys Eng Sci Med. 2015;38:7-22.

23. Hill-Kayser CE, Plastaras JP, Tochner Z, Glatstein E. TBI during BM and SCT: review of the past, discussion of the present and consideration of future directions. Bone Marrow Transplant. 2011;46:475-484.

24. Locatelli F, Lucarelli B, Merli P. Current and future approaches to treat graft failure after allogeneic hematopoietic stem cell transplantation. Expert Opin Pharmacother. 2014;15:23-36.

25. Holtick U, Albrecht M, Chemnitz JM, et al. Bone marrow versus peripheral blood allogeneic haematopoietic stem cell transplantation for haematological malignancies in adults. Cochrane Database Syst Rev. 2014; CD010189.

26. Penack O, Becker C, Buchheidt D, et al. Management of sepsis in neutropenic patients: 2014 updated guidelines from the Infectious Diseases Working Party of the German Society of Hematology and Medical Oncology (AGIHO). Ann Hematol. 2014;93:1083-1095.

27. Bodei L, Kidd M, Paganelli G, et al. Long-term tolerability of PRRT in 807 patients with neuroendocrine tumours: the value and limitations of clinical factors. Eur J Nucl Med Mol Imaging. 2015;42:5-19. 